\section{Baculovirus Expression Vectors that Incorporate the Foreign Protein into Viral Occlusion Bodies}

BioTechniques 34:81-87 (January 2003)

\begin{abstract}
Current baculovirus expression systems typically produce soluble proteins that accumulate within the infected insect cell or are secreted into the growth medium. A system has now been developed for the incorporation of foreign proteins, along with the matrix protein, polyhedrin, into baculovirus occlusion bodies. Initial studies showed that a recombinant virus expressing a translational fusion between polyhedrin and GFP did not form occlusion bodies. However, a baculovirus coexpressing native polyhedrin and
\end{abstract}

the polyhedrin-GFP fusion protein formed occlusion bodies that fluoresced under UV light, demonstrating that they included the polyhedrin-GFP fusion protein. This was confirmed by immunoblot analysis. Thus, incorporation of a foreign protein into occlusion bodies depends on an interaction between native polyhedrin and the polyhedrin fusion protein. Electron microscopy demonstrated that the occlusion bodies containing GFP also incorporated virions as expected. These ColorPol occlusion bodies were as infectious to insect larvae as occlusion bodies produced by wild-type virus. This new system expands the capabilities for foreign gene expression by baculoviruses, which has implications for biopesticide design, novel vaccine delivery systems, and fusion protein purification applications.

\section{INTRODUCTION}

Baculoviruses are a large family of viruses that mostly infect lepidopteran insects. A characteristic feature of baculoviruses is the formation of large proteinaceous occlusion bodies, called polyhedra, in which the virions are embedded. Polyhedra protect the virus in the environment and are required for horizontal spread between hosts. They can be up to $15 \mu \mathrm{m}$ in diameter. The matrix of these bodies is composed largely of the protein polyhedrin. This is highly abundant, comprising up to $50 \%$ of the protein in an infected cell $(8,13,14)$. Several studies have examined factors affecting polyhedra morphogenesis $(2-5,7,17)$. However, many aspects of occlusion body assembly remain unclear.

Baculoviruses are of considerable interest because of their potential as biological insecticides and their use as eukaryotic gene expression vectors (12). Their development as an expression vector system was prompted by the fact 
that the polyhedrin gene is both highly expressed and nonessential for viral replication in cell culture. Thus, early expression vector systems typically involved the replacement of the polyhedrin gene with the gene of interest placed under the control of the polyhedrin promoter. This gave rise to recombinant viruses that overproduced the foreign protein in cell culture but that could not produce occlusion bodies. More recently, expression vector systems have been developed that generate recombinant viruses that are able to produce occlusion bodies (13). Such viruses are particularly useful for producing the foreign protein in infected insects.

A wide variety of variants of these basic systems has been developed that allows the use of different promoters or provide more rapid screening systems for the identification of the recombinant viruses. Typically, the foreign protein is produced in a soluble form and is directed to its appropriate subcellular or extracellular location. Where the virus is capable of producing occlusion bodies, these are normal viral polyhedra and do not contain the foreign protein. However, there are circumstances where it could be advantageous to incorporate a foreign protein directly within the polyhedrin matrix to produce recombinant polyhedra. For example, it could be useful to engineer polyhedra to carry an insect toxin for improved biopesticidal activity or to display foreign proteins as a vaccine delivery tool for vertebrates (6). An early study by McLinden et al. (11) demonstrated that the polyhedrin protein could be manipulated to encode small epitopes of foreign proteins. However, the number and the location of these additional amino acids affected whether successful assembly of recombinant polyhedra occurred.

Here we describe the production of a recombinant baculovirus in which a foreign protein, GFP, is actually incorporated into viral polyhedra. Polyhedra that incorporate this full-length protein retain the ability to occlude virions. These occlusion bodies are infectious to insects and fluoresce under UV light, demonstrating that GFP remains functional. This technology expands the range of applications of the baculovirus expression system.

\section{MATERIALS AND METHODS}

\section{Cell Lines and Viruses}

Spodoptera frugiperda IPLB Sf21$\mathrm{AE}$ (16) clone 9 (Sf9) cells were maintained at $27^{\circ} \mathrm{C}$ in $\mathrm{TC} 100$ medium supplemented with $10 \%$ heat-inactivated FBS. All viruses were propagated and titered in Sf9 cells $(13,14)$. Polyhedra and viral DNA were obtained from $\mathrm{Sf} 9$ cells by standard methods $(13,14)$.

\section{Recombinant Transfer Vectors}

The 730-bp GFP gene from pGFP (BD Biosciences Clontech, Palo Alto, CA, USA) was inserted into the BamHI and PstI sites of pBacPAK8 (BD Biosciences Clontech) to yield pAcG (Figure 1A). A 903-bp region from AcMN$\mathrm{PV}$ polyhedrin was amplified using the primers 5'-GCCATTGTAATGAGACGCAC- $3^{\prime}$ and $5^{\prime}$-AACTCGAGATACGCCGGACCAGTGAAC-3'. The latter replaces the termination codon TAA with an XhoI site (underlined). All PCRs comprised 35 cycles of $94^{\circ} \mathrm{C}$ for $1 \mathrm{~min}$, $55^{\circ} \mathrm{C}$ for $1 \mathrm{~min}$, and $72^{\circ} \mathrm{C}$ for $1 \mathrm{~min}$. The product was digested with $E c o$ RV and XhoI and inserted into pBacPAK8 to yield pBacPAK-AcPOL. GFP was amplified from $\mathrm{pGFP}$ using the primers
5'-AACTCGAGATGAGTAAAGGAGAAGAAC-3' and 5'-CTATTTGTATAGTTCATCCATGCC- $3^{\prime}$. The amplified product was blunt-ended with Klenow, digested with XhoI site (underlined), and inserted into XhoI- and SmaIcut $\mathrm{pBacPAK}-\mathrm{AcPOL}$ to yield $\mathrm{pAcPG}$ (Figure 1B). To construct pColorPol, a 1605 -bp fragment of the polyhedrinGFP fusion from $\mathrm{pAcPG}$ was amplified with 5'-AAAAAAAAGATCTACCATGCCGGATTATTC- $3^{\prime}$ and $5^{\prime}$-CGCTCTAACATACCACCC-3' corresponding to the translational start and $3^{\prime}$ untranslated regions of polyhedrin. The amplified fragment was digested with $B g l \mathrm{II}$ (underlined) and SnaBI and inserted into BamHI- and SnaBI-cut pAcUW31 (BD Biosciences Clontech), giving pAcUWPG. AcMNPV polyhedrin was amplified with 5'-AAAAAAAAGATCTACCATGCCGGATTATTC- $3^{\prime}$ and $5^{\prime}$-GTAGAATTCATTAATACGCCGGACCAG-3'. These include BglII and EcoRI sites (underlined). The amplified gene was cloned into pAcUW-PG to give pColorPol (Figure 1C).

\section{Construction of Recombinant Viruses}

One microgram of BacPAK6 viral DNA (BD Biosciences Clontech) and 5

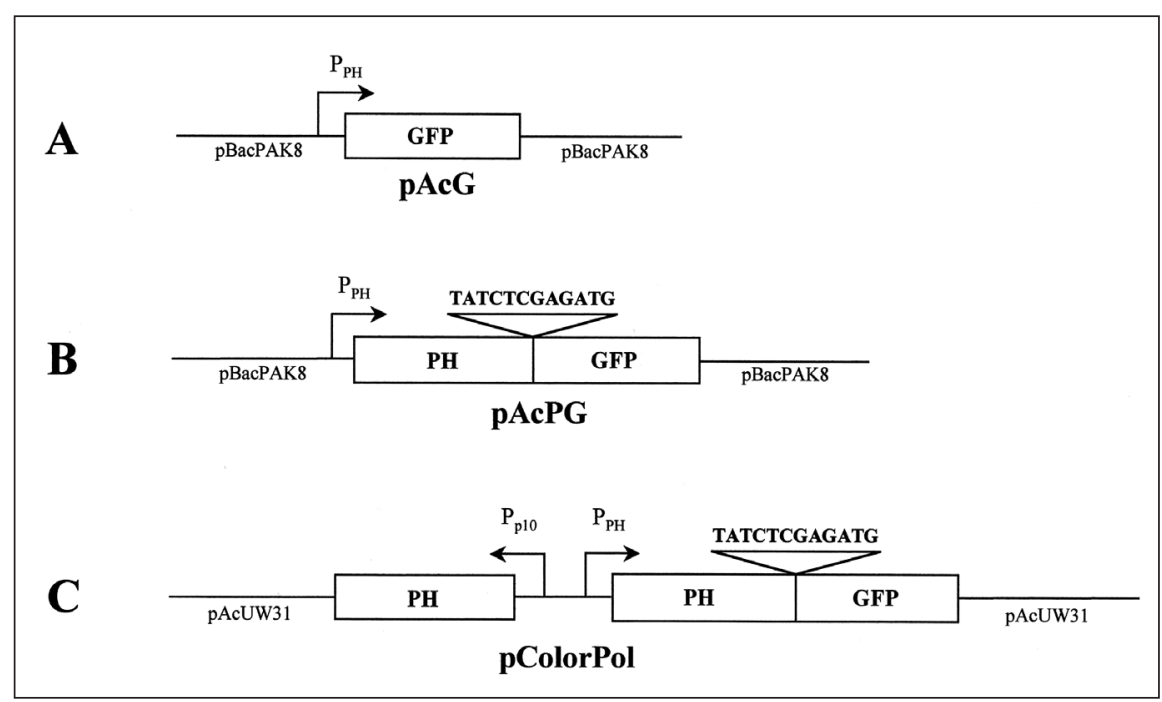

Figure 1. Transfer vectors for the production of recombinant baculoviruses. (A) The transfer vector pAcG was constructed by insertion of the GFP gene (GFP) into pBacPAK8, under the control of the polyhedrin promoter $\left(\mathrm{P}_{\mathrm{PH}}\right)$. (B) The transfer vector $\mathrm{pAcPG}$ was constructed by insertion of the GFP gene as a translational fusion at the $3^{\prime}$ end of the AcMNPV polyhedrin gene $(\mathrm{PH})$, under the control of the polyhedrin promoter $\left(\mathrm{P}_{\mathrm{PH}}\right)$. The transfer vector $\mathrm{pAcUW} 31$ was used for the construction of the transfer vector pColorPol $(\mathrm{C})$. The AcMNPV polyhedrin gene and the fusion gene polyhedrin-GFP were introduced under the control of $\mathrm{p} 10\left(\mathrm{P}_{\mathrm{p} 10}\right)$ or polyhedrin $\left(\mathrm{P}_{\mathrm{PH}}\right)$ promoters, respectively. The arrows show the direction of transcription. 


\section{Short Technical Reports}

$\mu \mathrm{g}$ transfer vector DNA were cotransfected into Sf9 cells by lipofection as described (13). The virus-containing supernatant was harvested five days later, and recombinant AcMNPV was isolated by plaque purification $(13,14)$. The genome structures of the recombinant viruses were confirmed by PCR, restriction endonuclease analysis, and Southern blotting (data not shown).

\section{SDS-PAGE and Immunoblot Analysis}

Sf9 cells were mock-infected or infected with wild-type or recombinant AcMNPV at an MOI of $5 \mathrm{pfu} / \mathrm{cell}$, and harvested three days post-infection. Total cellular lysates were subjected to $10 \%$ SDS-PAGE (9), electroblotted, and incubated with anti-GFP (BD Bio-

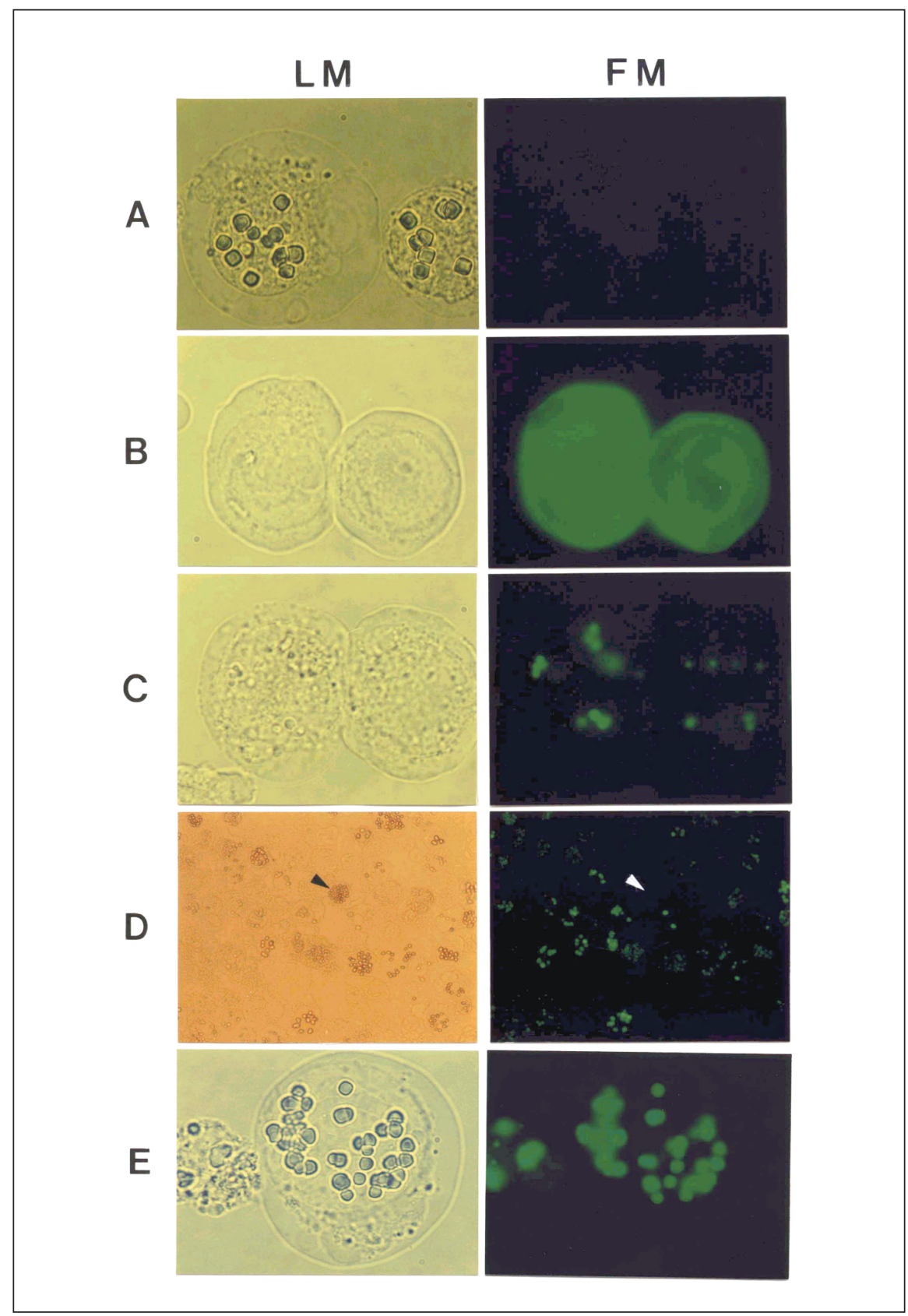

Figure 2. Polyhedra formation by AcMNPV recombinants. Sf9 cells infected with wild-type AcMNPV $(A, \times 1000), A c G(B, \times 1000)$, AcPG $(C, \times 1000)$, both wild-type AcMNPV and AcPG (D, $\times 200)$, or ColorPol $(\mathrm{E}, \times 1000)$ were observed four days post-infection by light (LM) and fluorescent (FM) microscopy. Arrows in panel D indicate non-fluorescent polyhedra.

sciences Clontech) or anti-AcMNPV polyhedrin antibody. The anti-AcMNPV polyhedrin antibody was generated in mice by injection of purified AcMN$\mathrm{PV}$ polyhedrin protein. Bound antibodies were visualized by incubation with goat anti-mouse IgG conjugated with alkaline phosphatase [1:1000 (v/v); Sigma, St. Louis, MO, USA) as described previously (15).

\section{Immunogold Staining}

Samples were fixed for $3 \mathrm{~h}$ in $4 \%$ formaldehyde, $1 \%$ glutaraldehyde in $0.1 \mathrm{M}$ sodium phosphate ( $\mathrm{pH} 7.5)$ containing $0.15 \mathrm{mM} \mathrm{CaCl}_{2}$ and $0.45 \mathrm{M}$ sucrose (FM), and incubated overnight in FM, pH 10.4, without glutaraldehyde. The samples were rinsed in $0.1 \mathrm{M}$ sodium phosphate ( $\mathrm{pH} 7.5$ ), dehydrated in a graded ethanol series (up to $95 \%$ ), and embedded in Lowicryl K4M (Polysciences, Warrington, PA, USA). Ultra-thin sections mounted on formvar-coated nickel grids were treated for $10 \mathrm{~min}$ with TBS $(0.02 \mathrm{M}$ Tris$\mathrm{HCl}, \mathrm{pH} 7.5$, containing $0.5 \mathrm{M} \mathrm{NaCl}$ ). The sections were etched with $3 \%$ $\mathrm{H}_{2} \mathrm{O}_{2}$ in double-distilled water for 5 min and blocked with 3\% BSA in TBS for $30 \mathrm{~min}$. Double immunogold staining was performed as described previously (1). The sections were incubated with primary antiserum against GFP (diluted 1:200), washed in TBST three times for $15 \mathrm{~min}$ with gentle agitation, and exposed to gold-conjugated goat anti-mouse $\operatorname{IgG}(30 \mathrm{~nm}$; BioCell, Cardiff, UK) diluted 1:10 in TBST for $60 \mathrm{~min}$. After washing with $0.3 \%$ BSA in TBS, the grids were dried, and the reverse of the grid was incubated with antiserum against polyhedrin $(1: 300)$ and gold-conjugated goat anti-mouse IgG (10 nm; Zymed, CA, USA) diluted 1:30 in TBST.

\section{Bioassays}

The infectivity and virulence of ColorPol were compared to wild-type AcMNPV in the Diamondback moth, Plutella xylostella. P. xylostella larvae were obtained from a laboratory colony and reared at $25^{\circ} \mathrm{C}$ on Chinese cabbage under a $16 \mathrm{~h}: 8 \mathrm{~h}$ light dark cycle. To determine the median lethal dose $\left(\mathrm{LD}_{50}\right)$, 128 third-instar larvae were fed six dos- 
Table 1. LD $_{50}$ and $\mathrm{ST}_{50}$ Values of ColorPol and Wild-Type AcMNPV against $P$. xylostella Larvae

\begin{tabular}{|c|c|c|c|c|c|c|}
\hline \multirow[b]{3}{*}{ Virus } & \multicolumn{3}{|c|}{$L^{L 0}$} & \multicolumn{3}{|c|}{$\mathrm{ST}_{50}$} \\
\hline & \multicolumn{3}{|c|}{ Polyhedra/ } & \multirow[b]{2}{*}{$n^{*}$} & \multirow[b]{2}{*}{ Hours } & \multirow[b]{2}{*}{$95 \% \mathrm{Cl}$} \\
\hline & $n^{\star}$ & Larva & $95 \% \mathrm{Cl}$ & & & \\
\hline AcMNPV & 768 & 2798 & $1483-4884$ & 128 & 92.8 & $88.0-98.0$ \\
\hline ColorPol & 768 & 4907 & $3143-8366$ & 128 & 108.7 & $105.5-112.3$ \\
\hline \multicolumn{4}{|c|}{ *Number of insects } & & & \\
\hline
\end{tabular}

es $(20480,10240,5120,1280$, and 640 polyhedra/larva) of AcMNPV or ColorPol on a small plug of Chinese cabbage leaves. Larvae that consumed the dose in $24 \mathrm{~h}$ were transferred to fresh Chinese cabbage leaves and examined twice daily. Cadavers were removed, and the cause of death was confirmed. The data were analyzed by probit analysis. To determine the median survival time $\left(\mathrm{ST}_{50}\right), 128$ third-instar larvae were fed 10240 polyhedra/ larva of wild-type AcMNPV or ColorPol (approximately equivalent to an $\mathrm{LD}_{90}$ dose) on a small plug of Chinese cabbage leaves. After feeding, the larvae were transferred to fresh diet and observed as before. ST calculations were made using the vistat program (Boyce Thompson Institute, Ithaca, NY, USA). The $95 \%$ confidence intervals $(\mathrm{CI})$ were also calculated for the $\mathrm{LD}_{50}$ and $\mathrm{ST}_{50}$ values.

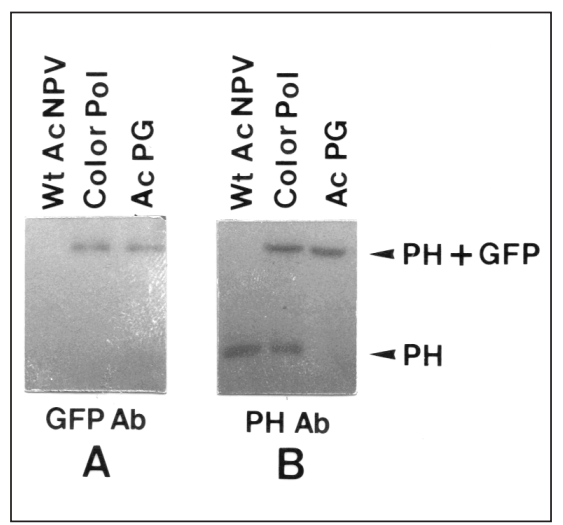

Figure 3. Immunoblot analysis of Sf9 cells infected with ColorPol. At three days post-infection, total cellular lysates were separated by $10 \%$ SDS-PAGE and immunoblotted with anti-GFP (A) or anti-polyhedrin (B) antibody. Wild-type AcMNPV and AcPG served as controls. The positions of polyhedrin $(\mathrm{PH})$ and the polyhedrinGFP fusion protein $(\mathrm{PH}+\mathrm{GFP})$ are indicated.

\section{RESULTS}

\section{Formation of Polyhedra Incorporating GFP}

The structures of the transfer plasmids used to generate the recombinant viruses employed in this study are summarized in Figure 1. The virus AcG was generated by cotransfection of parental Autographa californica nucleopolyhedrovirus (AcMNPV) with the transfer plasmid pAcG (Figure 1A) and therefore expresses GFP in place of polyhedrin under the control of the polyhedrin promoter. This represents the classical approach to the generation of a baculovirus expression vector. As expected, AcG did not form polyhedra, but GFP was detected in a soluble form in the nucleus and cytoplasm of infected cells (Figure 2B). The first approach we attempted to generate a virus that indicated. The bars represent $1 \mu \mathrm{m}$. produces occlusion bodies incorporating GFP was to express a polyhedrinGFP fusion protein. The transfer plasmid pAcPG includes the GFP gene fused in frame to the $3^{\prime}$ end of the polyhedrin gene (Figure 1B). The fusion protein is under the control of the polyhedrin promoter. However, the recombinant virus generated, AcPG, still did not form polyhedra, unlike wild-type viral infection (Figure 2A). GFP was now detected exclusively in the nucleus, apparently in a particulate form, but these particles were distinct from polyhedra (Figure 2C).

We reasoned that the formation of normal occlusion bodies might require the presence of unfused polyhedrin protein. Thus, we tried coinfecting cells with both wild-type AcMNPV and AcPG. In this case, occlusion body formation was observed (Figure 2D). In most cells, the polyhedra were fluorescent under UV light, indicating incorporation of GFP. A small proportion of cells contained non-fluorescent polyhedra. Cells coinfected with wild-type AcMNPV and AcG produced non-fluorescent polyhedra (data not shown). We suspected that in the AcMNPV-AcPG coinfection (Figure 2D) a few cells had been infected with wild-type AcMNPV only, producing non-fluorescent polyhedra, whereas most cells had been infected with both viruses and produced

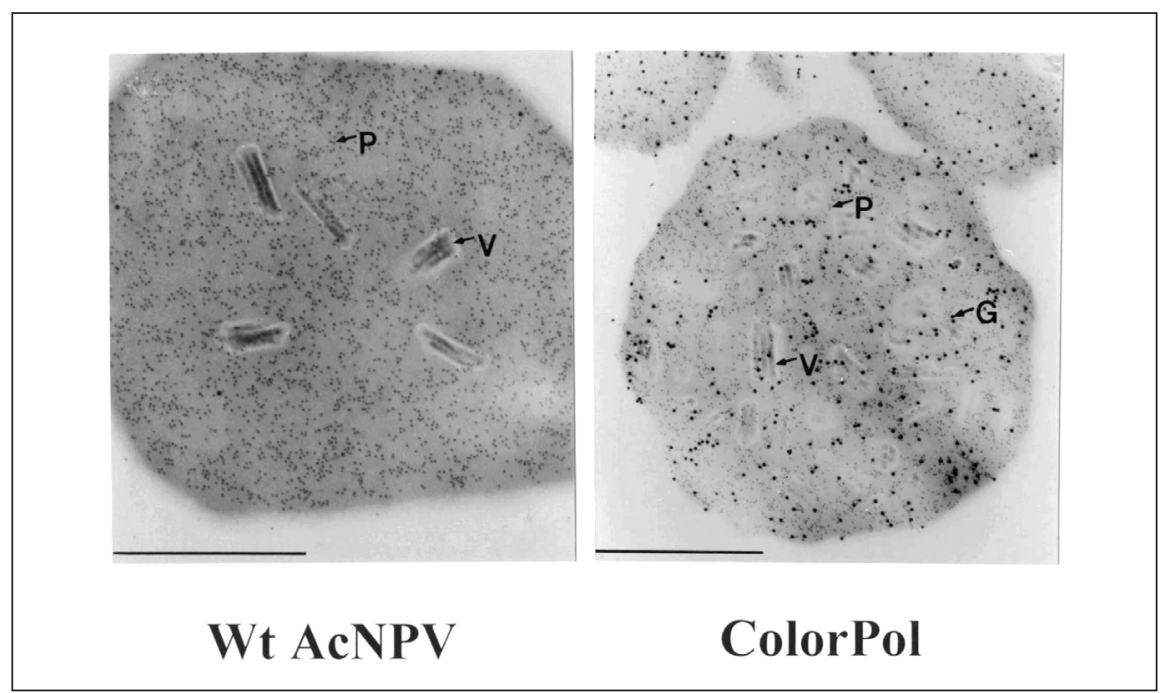

Figure 4. Immunogold labeling of recombinant polyhedra. Purified polyhedra formed by wild-type AcMNPV or ColorPol (left and right panels, respectively) were analyzed by immunogold labeling with a mixture of antisera against polyhedrin and GFP. Gold particles attached to the anti-polyhedrin (P, $10 \mathrm{~nm})$ and anti-GFP (G, $30 \mathrm{~nm})$ antibodies were observed by transmission electron microscopy. Virions (V) are 


\section{Short Technical Reports}

fluorescent polyhedra. This suggested that some native polyhedrin protein is required for occlusion body formation. Therefore, we constructed the virus ColorPol which coexpresses native polyhedrin and the polyhedrin-GFP fusion (Figure 1C). In this virus, the polyhedrin-GFP fusion protein is expressed under the control of the polyhedrin promoter, whereas the $\mathrm{p} 10$ promoter drives the polyhedrin expression. P10 is another abundantly produced baculovirus protein that is expressed with very similar kinetics to the polyhedrin gene (13). All ColorPol-infected cells produced fluorescent polyhedra (Figure 2E).

\section{Characterization of Polyhedra Displaying GFP}

Protein synthesis in wild-type AcMNPV-, ColorPol-, and AcPG-infected Sf9 cells was analyzed by immunoblotting (Figure 3). Both polyhedrin and
GFP antisera detected the polyhedrinGFP fusion as a $60-\mathrm{kD}$ a protein in ColorPol- and AcPG-infected cell lysates. The polyhedrin antibody detected polyhedrin in ColorPol- and wild-type AcMNPV-infected cells. Purified ColorPol polyhedra also comprised the fusion protein and native polyhedrin (data not shown). To verify the presence of GFP in ColorPol polyhedra, occlusion bodies were examined by immunogold labeling (Figure 4). Labeling due to polyhedrin and GFP was homogeneously distributed throughout the occlusion bodies. These data also showed that the occlusion bodies are morphologically normal and contained virions, suggesting that they might be infectious. This was confirmed by bioassay of ColorPol occlusion bodies fed to $P$. xylostella larvae. The infectivity of the ColorPol virus was not significantly different from that of wild-type AcMN$\mathrm{PV}$, but insects infected by ColorPol displayed a $17 \%$ increase in survival time (Table 1). PCR analysis of ColorPol virus recovered from infected insects confirmed the recombinant locus is stable (data not shown).

\section{DISCUSSION}

Our initial attempt to produce occlusion bodies displaying GFP entailed the generation of a recombinant virus expressing a polyhedrin-GFP fusion protein. The fusion protein was detected exclusively in the nucleus, whereas GFP alone was present throughout the cell. Thus, sequences in polyhedrin are responsible for the retention of the fusion protein in the nucleus. Nuclear localization signals in polyhedrin have been described previously (7). The polyhedrin-GFP fusion protein did not form occlusion bodies but was detected in a particulate form, suggesting that 
some assembly had occurred. McLinden et al. (11) reported that polyhedrin proteins with short insertions in the central part of the protein either failed to form occlusion bodies or gave rise to abnormal occlusion bodies. Insertion of the foreign amino acids at the N-terminus gave rise to morphologically normal occlusion bodies that did not occlude virus efficiently.

We chose a different strategy of coexpressing native polyhedrin with the fusion protein. This virus, ColorPol, coexpresses native polyhedrin and a polyhedrin fusion protein. ColorPol occlusion bodies are as infectious to insects as their wild-type counterparts, indicating that the incorporation of GFP does not affect dissolution of the occlusion bodies in the gut of the insect. Surprisingly, the speed of kill of the virus seemed to be somewhat slower than that of wild-type virus (Table 1). It is not clear whether this is due to alterations in the viral polyhedra or to the presence of GFP in the infected cells.

A major application of recombinant baculovirus technology has been to produce viruses with improved insecticidal properties. The technology we have described facilitates the development of recombinant baculoviruses that can deliver insecticidal proteins that are active in the gut of the insect. We have recently exemplified this approach by the generation of a recombinant virus that produces occlusion bodies containing an insecticidal Bacillus thuringiensis crystal protein toxin (ColorBtrus). This was based on the construction of a polyhedrin-Bt toxin-GFP fusion polypeptide (8). Both the infectivity and speed of action of ColorBtrus occlusion bodies are dramatically enhanced compared to wild-type virus (Je et al., submitted).

This technology should also facilitate other novel applications of baculovirus expression vectors. For example, it should represent an advantageous approach to the expression of proteins to be used as subunit vaccines. The delivery of immunogens in a particulate form should result in the development of a robust inflammatory $\left(\mathrm{T}_{\mathrm{H} 1^{-}}\right.$ mediated) response in vertebrates. The presence of other viral proteins in the occlusion body will also facilitate this. The ability to incorporate simultane- ously more than one protein into the polyhedra, as we have done for ColorBtrus, raises the possibility of using this approach for the delivery of multivalent subunit vaccines or for the delivery of immune regulatory proteins along with the immunogen. Another possible application of this technology is the production of insoluble bait protein for the capture of interacting proteins by panning. We are currently exploring these and other applications of the technology.

\section{REFERENCES}

1.Bendayan, M. 1982. Double immunocytochemical labeling applying the protein A-gold technique. J. Histochem. Cytochem. 30:81-85.

2.Carstens, E.B., A. Krebs, and C.E. Gallerneault. 1986. Identification of an amino-acid essential to the normal assembly of Autographa californica nuclear polyhedrosis virus polyhedra. J. Virol. 58:684-688.

3.Carstens, E., G. Williams, P. Faulkner, and S. Partington. 1992. Analysis of polyhedra morphology mutants of Autographa californica nuclear polyhedrosis virus: molecular and ultrastructural features. J. Gen. Virol. 73:1471-1479.

4.Carstens, E.B., L.B. Ye, and P. Faulkner. 1987. A point mutation in the polyhedrin gene of a baculovirus Autographa californica MNPV prevents crystallization of occlusion bodies. J. Gen. Virol. 68:901-906.

5.Duncan, R., K.L. Chung, and P. Faulkner. 1983. Analysis of a mutant of Autographa californica nuclear polyhedrosis virus with a defect in the morphogenesis of the occlusion body macromolecular lattice. J. Gen. Virol. 64:1531-1542.

6.Fraser, M.J., E.D. Rosen, and V.A. Ploplis, inventors; American Biogenetic Sciences, assignee. Recombinant baculovirus occlusion bodies in vaccines and biological insecticides. US patent no. 4870023.1988 Feb 8 .

7.Jarvis, D., D. Bohlmeyer, and A. Garcia. 1991. Requirements for nuclear localization and supramolecular assembly of a baculovirus polyhedrin protein. Virology 185:795-810.

8.Kang, S.K., Y.H. Je, B.R. Jin, H.W. Park, J.Y. Roh, and J.H. Chang, inventors. Recombinant baculovirus, construction method thereof and insect pesticidal composition containing the same. US patent no. 6338846 1998 Nov 6

9.Laemmli, U.K. 1970. Cleavage of structural proteins during the assembly of the head of bacteriophage T4. Nature 227:680-685.

10.Maeda, S. 1989. Gene transfer vectors of a baculovirus, Bombyx mori nuclear polyhedrosis virus, and their use for expression of foreign genes in insect cells, p. 167-182. In J. Mitsuhashi (Ed.), Invertebrate Cell System Applications, Vol. 1. CRC Press, Boca Raton, FL.

11.McLinden, J.H., V.A. Ploplis, M.J. Fraser, and E.D. Rosen. 1992. Expression of foreign epitopes on recombinant occlusion bodies of baculoviruses. Vaccine 10:231-237.

12.Miller, L.K. 1997. The Baculoviruses. Plenum Press, New York.

13.O'Reilly, D.R., L.K. Miller, and V.A. Luckow. 1992. Baculovirus Expression VectorsA Laboratory Manual. Oxford University Press, New York.

14.Summers, M.D. and G.E. Smith. 1987. A manual of methods for baculovirus vectors and insect cell culture procedures. Texas Agricultural Experiment Station Bulletin 1555.

15.Towbin, H., T. Staehelin, and J. Gordon. 1979. Electrophoretic transfer of proteins from polyacrylamide gels to nitrocullulose sheets: procedure and some applications. Proc. Natl. Acad. Sci. USA 76:4350-4354.

16.Vaughn, J.L., R.H. Goodwin, G.J. Tompkins, and P. McCawley. 1977. The establishment of two cell lines from the insect Spodoptera frugiperda (Lepidoptera: Noctuidae). In Vitro 13:213-217.

17.Woo, S.D., W.J. Kim, H.S. Kim, B.R. Jin, Y.H. Lee, and S.K. Kang. 1998. The morphology of the polyhedra of a host range-expanded recombinant baculovirus and its parents. Arch. Virol. 143:1209-1214.

This research was supported in part by the Brain Korea 1 Project. S.K.K. is deceased. Address correspondence to Dr. Yeon Ho Je, School of Agricultural Biotechnology Seoul National University, Suwon 441744,Korea.e-mail:btrus@hanmail.net

Received 18 June 2002; accepted 10 October 2002.

Y.H. Je' ${ }^{1,4}$, B.R. Jin ${ }^{2}$, H.W. Park $^{1}$, J.Y. Roh ${ }^{1}$, J.H. Chang ${ }^{1}$, S.J. Seo ${ }^{3}$, J.A. Olszewski ${ }^{4}$, D.R. O'Reilly $^{4}$, and S.K. Kang ${ }^{1}$

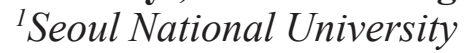
Suwon, Korea

${ }^{2}$ Dong-A University

Pusan, Korea

${ }^{3}$ Gyeongsang National

University

Jinju, Korea

${ }^{4}$ Imperial College of Science, Technology, and Medicine

London, UK

For reprints of this or any other article, contact Reprints@BioTechniques.com 